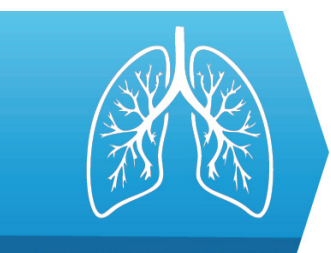

PNEUMOLOGY

\title{
The impact of diabetes mellitus on the anti- tuberculosis treatment outcome: a series of cases from the Republic of Moldova
}

Evelina Lesnic, Alina Malic, Ovidiu Tafuni

Department of Pneumophthisiology, Nicolae Testemițanu State Medicine and Pharmacy University, Republic of Moldova
DOI: $10.15386 / \mathrm{mpr}-1974$

Manuscript received: 22.07 .2020

Received in revised form: 17.11.2020

Accepted: 10.12.2020

Address for correspondence:

evelina.lesnic@usmf.md

This work is licensed under a Creative Commons Attribution-NonCommercialNoDerivatives 4.0 International License

\begin{abstract}
Background and aims. The Republic of Moldova ranks among the European Region countries with the highest global incidence of tuberculosis. Almost $5 \%$ of the cases with pulmonary TB had been diagnosed with DM in 2017. The aim of this study was to assess the impact of diabetes mellitus on the anti-tuberculosis treatment effectiveness for the improvement of the disease outcome.

Methods. A retrospective, longitudinal and case-control study, which included a total number of 252 patients diagnosed with pulmonary tuberculosis and distributed into a study group of 93 patients diagnosed with diabetes mellitus and a control group of 159 patients without glycemic disorders was performed. The statistical analysis was performed using EpiInfo software. The statistical analysis of the differences between normally distributed continuous variables was tested with the Student T-test.

Results. Based on the collected data we established that the majority of the patients with glycemic disorders were diagnosed with the type 2 diabetes, associated with hyperglycemia and in half of them complications of diabetes were revealed. The age older 55 years and the low economical state were common characteristics of the diabetic patients. Tuberculosis was detected in every second diabetic patient through the radiological screening compared with the passive detection of most of the nondiabetic patients. The anti-tuberculosis treatment outcome was endangered by a higher rate of the adverse drug events in patients with diabetes, which contributed to death in $15 \%$, lost to follow-up $7 \%$, and therapeutic failure in $6 \%$.

Conclusions. The anti-tuberculous treatment outcome in patients diagnosed with tuberculosis and diabetes mellitus was significantly diminished by glycemic disorders, history of the previous anti-tuberculous treatment and adverse drug reactions. Individualized therapeutic approach to tuberculosis could improve the treatment effectiveness.
\end{abstract}

Keywords: tuberculosis, diabetes, risk factors, outcome

\section{Introduction}

The Republic of Moldova ranks among the European Region countries with the highest global incidence of tuberculosis (TB) - 83.3/100 000 population in 2017 and among the countries with the highest level of multidrug-resistant tuberculosis (MDRTB) [1]. In 2017, 26.6\% of new cases and $63.8 \%$ of previously treated cases were diagnosed with MDR-TB in the Republic of Moldova [2]. The current policy of the World Health Organization (WHO) on tuberculosis control is based on the End TB strategy (Strategy) [3]. The general goal of the Strategy is to end the global TB epidemic. The milestones of the Strategy for 2025 are: to reduce TB deaths by $75 \%$ compared with 2015 , to reduce the global TB incidence (incidence $<55 / 100000$ population) by $50 \%$, and zero families facing catastrophic costs due to TB [3].

Diabetes mellitus (DM) is an important public health issue, one of the four priority non-communicable diseases targeted by the sustainable 
development goals (SDG) [4]. SDG target 3.4 was assessed by the indicator 3.4.1 that counts on the reduction of premature deaths between the ages of 30 and 70 years from cardiovascular disease, cancer, DM and chronic respiratory disease [4]. Diabetes is a group of metabolic diseases characterized by hyperglycemia resulting from the disturbances in insulin secretion, insulin action or both [5]. The chronic hyperglycemia contributes to the long-term damage, dysfunction of different organs, increased risk for infections of lower respiratory tract and TB [6]. The risk for TB is increased 1.5-7.8 times in patients with uncontrolled hyperglycemia due to high levels of pro-inflammatory mediators (IL- $6, \mathrm{TNF}-\alpha$ ) released by the infection with Mycobacterium tuberculosis in the lung tissue [7]. In the Republic of Moldova $12.3 \%$ of the population suffer from impaired glucose tolerance. Almost $5 \%$ of cases of pulmonary TB had been diagnosed with DM in 2017 [9]. The mortality caused by DM was $11.5 / 100000$ population in 2017 [8]. The patients diagnosed with DM are annually screened for TB and the rate of diabetic patients detected through the active way achieved $65 \%$ in 2017 [9]. The most important step in the control of TB is the adequate treatment, according to the drug resistance profile. The objectives of the anti-tuberculous treatment constitute: (1) to cure the patient; (2) to prevent the death from active TB or its late effects; (3) to prevent relapse; (4) to decrease the risk of the mycobacteria transmission to others; (5) to prevent the development of the acquired MDR-TB [10].

The standard regimen for drug-susceptible TB includes the first-line drugs: Isoniazid, Rifampicin, Pyrazinamide and Streptomycin or Ethambutol $[9,10]$. The treatment of patients with diabetes is frequently a challenge and the evolution is unfavorable. The sputum conversion is delayed, the rate of therapeutic failure is increased 7 times and the mortality 6 times $[11,12]$. A cluster of risk factors is involved in the patients' poor evolution: the glucose metabolism disorders during the anti-tuberculous treatment, the high rate of the adverse drug reactions and acquired MDR-TB due to the low absorption of the drugs from the gastrointestinal tract [13].

The rate of the adverse anti-tuberculous drug effects in diabetic patients ranges between $20 \%$ and $54 \%$, according to the applied therapeutic regimen [1316]. Uncontrolled hyperglycemia affects multiple organs, such as eyes, liver, kidney, nerves, and cardiovascular system $[5,8]$. The most frequent side effects are: peripheral neuritis caused by Isoniazid, toxic hepatitis determined by Isoniazid, Rifampicin, Pyrazinamide, renal toxicity of aminoglycozides (Streptomycin, Kanamycin, Amikacin) and optic neuritis due to Ethambutol [9]. As a consequence, the hospitalization of the patients with DM is recommended for establishing the clinical tolerance to the treatment [9].

Drug interaction is confirmed in patients taking many drugs. There are numerous potential drug interactions clinical undetectable [17]. Most of the drug-drug interactions in patients treated with anti-tuberculous drugs will be due to the enzyme inducing effects. Rifampicin and Isoniazid, firstline drugs affects liver enzymes. Rifampicin is a potential liver enzyme inducer and Isoniazid acts as inhibitor. Rifampicin induces the metabolism of the antidiabetic drugs sulfonylureas and diminishes their concentration in the blood in 2 weeks after the onset of treatment. Rifampicin increases the absorption of the glucose from the gastro-intestinal tract and contributes to the transient hyperglycemia and glycosuria. Considering that insulin is not metabolized, it has no pharmacokinetic interaction with Rifampicin or other anti-tuberculous drugs. Furthermore, the insulin is recommended instead of the oral antidiabetic drugs, although the rate of hypoglycemic episodes is higher [17]. In severe forms of TB, the association of the insulin with metformin is recommended, for maintaining the control of the glycaemia [18]. Drug-drug interactions can occur when patients have also HIV and are treated with anti-HIV drugs, which are the substrates of metabolic or transporter liver enzymes [17].

Despite including the patients with DM in the risk groups for annual screening, they are regularly detected with severe forms of TB with chronic evolution [19]. Although usual localization is in the upper and posterior lung segments, in DM often appears in the segments III, IV and V, what contributes to the misdiagnosis [9]. The late detection and late onset of the anti-tuberculous therapy, dietary errors and inadequate antidiabetic treatment represent the causes of premature death in patients with DM and TB [5-16]. Considering all the above-mentioned data, the study has been performed with the aim to assess the impact of diabetes mellitus on the anti-tuberculous treatment effectiveness for improvement of the disease outcome.

\section{Material and methods}

The research was cross-sectional and retrospective. It included a series of 252 patients diagnosed with pulmonary TB during the period 1.1.2017-31.12.2017 in the Republic of Moldova.

The following inclusion criteria determined the selection of the patients in the research: age over 18 years old, TB diagnosed by a pulmonologist and signed informed consent. The patients were distributed into two groups: for the Study Group (SG) 93 patients were selected in which the inclusion criteria were the DM diagnosed by an endocrinologist. In the Control Group (CG) we selected 159 patients without DM or history of DM. The exclusion criteria from the CG were DM and glycemic disorders.

The diagnosis of pulmonary TB was established according to the criteria provided by the national policy [9]. The sputum examination by Ziehl-Neelsen staining, culture on Lowenstein-Jensen and liquid BACTEC media, and chest X-ray investigations were performed in every patient from both groups. 
The methods for detecting glycemic disorders and diagnosis of DM in patients of the SG were the capillary glycemia, venous blood plasma glycemia (FVPG) after 10-14 hour of fast and the glycated albumin $\left(\mathrm{HbA}_{1 \mathrm{c}}\right)$ [5]. The capillary glycemia was measured with a glucometer in a blood sample obtained from the fingertip. The FVPC was assessed through an enzymatic method (hexokinase activity) in venous blood plasma and was considered as a diagnosis criteria for diabetes if additional confirmed clinical data were available. The method for the evaluation of the glycated albumin used the albuminspecific proteinase, ketamine oxidase and serum albumin assay reagent. Glycated albumin was calculated, as the percentage of glycated albumin in the total albumin. The concentration of the $\mathrm{HbA}_{1 \mathrm{c}} \geq 6.5 \%(48 \mathrm{mmol} / \mathrm{mol})$ was a diagnostic tool for DM.

The protocol schedule included the following data about the patients:

1. Biological and social characteristics: sex (malefemale rate), age (distribution in age groups according to the WHO recommendations), demographic characteristics (urban/rural).

2. Economic background: economic status (employed, unemployed, retired, disabled) and healthinsurance coverage (presence/lack of health insurance). The disability was defined as the condition which limited the working capacity, allowing the patient to be supported by the state policy providing the financial support.

3. High-risk characteristics: homelessness, migration, history of detention, contact with TB patient.

4. Case-management: barriers to access to healthcare, method of the TB detection, medical staff which detected the TB.

5. Tuberculosis-related characteristics: localization (pulmonary/extrapulmonary), microbiological results (smear microscopy, culture on the conventional media, molecular-genetic tests and the drug susceptibility tests), comorbidities.

6. Glycemic control indicators: fasting venous plasma glucose (FVPG) and glycated hemoglobin (HbA1c) in patients with hyperglycemia.

7. Anti-tuberculous treatment, antidiabetic treatment and outcome.

The research was approved by the bioethics committee of "Nicolae Testemitanu" State University of Medicine and Pharmacy on 21 $1^{\text {th }}$ November 2017 and registered with the number 14 .

\section{Statistical analysis}

The statistical analysis was performed using EpiInfo software. The data were appreciated as nominal or quantitative. The frequency and percentage were reported for nominal data, and the mean and standard deviation were reported for continuous data. The statistical analysis of the differences between normally distributed continuous variables was tested with the Student T-test. A p-value of $<0.05$ was considered statistically significant. To estimate the strength of the association between the risk factors, the relative risk (RR) was used in the statistical analysis of the data.

\section{Results}

According to the type of glycemic disorders, in the SG, the type 1 diabetes was diagnosed in $17(18 \%)$ patients and type 2 - in $76(82 \%)$ patients. In $39(42 \%)$ patients from the SG different types of diabetes-related complications due to the peripheral vascular disease were diagnosed. The FVPG at the moment of the investigation for TB was assessed in all patients of the SG. The value of the FVPG exceeded the normal limit of $6 \mathrm{mmol} / \mathrm{L}$ in 68 (73\%) patients of the SG. Reporting to the total number of patients with hyperglycemia, in $31(46 \%)$ cases the FVPG was established in the range between 6.1 and 8 $\mathrm{mmol} / \mathrm{L}$, considered as a low increased indicator. In 18 $(26 \%)$ cases the value ranged between 8.1 and $12 \mathrm{mmol} / 1$ and was assessed as middle increased value. Higher than $12 \mathrm{mmol} / \mathrm{L}$ was detected in $19(28 \%)$ patients and was defined as uncontrolled hyperglycemia. Thus, according to the level of the glycemic disorders, we identified that in the majority of the cases the FVPG values exceeded the normal limit, demonstrating their susceptibility for acquiring tuberculosis. In 39 (42\%) cases the FVPG had ranged values, not exceeding the normal limit of $6,1 \mathrm{mmol} / \mathrm{L}$, but the long-term complications decreased the immune reactivity and contributed to acquiring tuberculosis.

The $\mathrm{HbA} 1_{c}$ concentration was determined in the patients with hyperglycemia, i.e. in $68(73 \%)$ of the SG. The $\mathrm{HbA} 1_{c}$ concentrations detected in the range between 4.8 and $5.6 \%$, considered as normal values, were found in 14 patients ( $20 \%$ of the investigated ones). Those patients were identified with low increased hyperglycemia. The values included in the range between 6 and $6.4 \%$ were found in $27(40 \%)$ patients. In $27(40 \%)$ cases the HbA1 concentrations exceeded $6.4 \%$ and in all cases the FVPG exceeded $12 \mathrm{mmol} / \mathrm{l}$.

The distribution of the patients according to the sex established a higher man/women rate in the CG (2.9) vs. SG (2.1). The distribution of the patients according to the age established that the young groups aged between 18 and 44 years prevailed in the CG - $100(63 \%)$ vs. 18 $(19 \%)$ in the SG. A similar rate of patients aged between 45 and 54 years was determined in both groups. The patients older than 55 years statistically predominated in the SG - $53(57 \%)$ vs. $22(14 \%)$ cases in the CG (Table I). Young individuals (less than 44 years old) prevailed in the CG and older than 55 years in those from the SG. Furthermore the patients' average age in the SG was $59 \pm 8$ years old, and in the $C G-38 \pm 5$ years old. 
Table I. Distribution of patients by sex and age.

\begin{tabular}{c|cc|c} 
Sex & Study group & Control group & \multirow{2}{*}{ P-value } \\
\cline { 2 - 3 } Age groups & $\mathbf{N = 9 3 ( P \% )}$ & $\mathbf{N = 1 5 9 ( P \% )}$ & \\
Men & $63(67)^{*}$ & $118(74)^{*}$ & $>0.05$ \\
Women & $30(33)$ & $41(26)$ & $>0.05$ \\
18-24 years & 0 & $20(13)$ & $<\mathbf{0 . 0 0 1}$ \\
25-34 years & $8(8)$ & $40(25)$ & $<\mathbf{0 . 0 0 1}$ \\
35-44 years & $10(11)$ & $40(25)$ & $<\mathbf{0 . 0 0 1}$ \\
45-54 years & $22(24)$ & $37(23)$ & $>0.05$ \\
55-64 years & $37(40)$ & $18(11)$ & $<\mathbf{0 . 0 0 1}$ \\
65+ years & $16(17)$ & $4(3)$ & $<\mathbf{0 . 0 0 1}$
\end{tabular}

Note: the applied statistical test: the paired sample T-test, P probability. *Absolute numbers and percentages per column (in brackets).

The distribution of the patients according to the economic status established that the employed persons contributing in this way to the health budget by paying taxes, health insurance policy and social taxes predominated in the CG - $60(38 \%)$ vs. $26(28 \%)$ cases in the SG, however, the statistical threshold was not achieved. Due to the predominance of elderly patients in the SG, the retired ones statistically prevailed. Since different complications of diabetes were diagnosed in $39(42 \%)$ cases from the SG, the rate of disabled individuals statistically predominated - $19(20 \%)$ compared to $3(2 \%)$ cases in the CG. Therefore, the low basic income associated with the retirement and disability statistically predominated in the SG - $27(29 \%)$ compared with $12(7 \%)$ cases in the CG. The distribution of the patients in economic categories established that the economically vulnerable, which included the unemployed, retired and disabled individuals predominated in the SG 68 (72\%) compared with 99 (62\%) in the CG (Table II). Thus, the economic vulnerability was confirmed as a risk factor for tuberculosis in patients with DM, RR $=1.38(95 \%$ CI: 1.15-2.12).

The patients' health insurance was analyzed. The insurance provided the free of charge screening procedures, both for TB and DM on the territory of the Republic of Moldova. The health insurance was revealed in a high proportion of both groups, in $61(66) \%$ cases of the SG vs. $72(45 \%)$ cases of the CG. Consequently, the lack of insurance, which predominated in the CG was considered a barrier to accessing the specialized healthcare (Table II).

Table II. Distribution by the economic groups.

\begin{tabular}{l|cc|c} 
Economic and & Study group & Control group & \multirow{2}{*}{ P-value } \\
\cline { 2 - 3 } insurance status & $\mathbf{N = 9 3 ( P \% )}$ & $\mathbf{N = 1 5 9 ( P \% )}$ & \\
Employed & $26(28)^{*}$ & $60(38)^{*}$ & $>0.05$ \\
$\begin{array}{l}\text { Disabled due to } \\
\text { disease }\end{array}$ & $19(20)$ & $3(2)$ & $<\mathbf{0 . 0 0 1}$ \\
Retired & $18(19)$ & $9(6)$ & $<\mathbf{0 . 0 0 1}$ \\
Unemployed & $30(32)$ & $87(55)$ & $<\mathbf{0 . 0 5}$ \\
$\begin{array}{l}\text { Lack of health } \\
\text { insurance }\end{array}$ & $32(44)$ & $87(55)$ & $>0.05$
\end{tabular}

Note: the applied statistical test: the paired sample T-test. *Absolute numbers and percentages per column (in brackets).
The demographic distribution identified that on average $50 \%$ of patients from both groups came from the urban localities of the Republic of Moldova. The patients residing in the rural areas of the country statistically predominated in the SG - $42(45 \%)$ compared with 38 (24\%) cases in the CG. Homeless or people without a place of permanent residence, neither urban nor rural, predominated in the CG - $39(25 \%)$ vs. $4(4 \%)$ patients in the SG. A similar rate of the economic migrants, who returned from abroad in the last 12 months were identified in both groups. A couple of patients from the CG had a life history of detention. The individuals from TB clusters were detected in a low proportion in both groups $-5(5 \%)$ in the SG and $15(9 \%)$ in the CG. Beside TB, in $71(76 \%)$ patients of the SG were diagnosed more than 2 associated diseases (comorbidities), among which one was DM. Complications of DM were diagnosed in $39(42 \%)$ patients from SG. In $24(15 \%)$ cases of the CG at least one associated with TB disease was diagnosed (Table III).

Table III. Distribution according to the demographic features.

\begin{tabular}{l|cc|c}
\multirow{2}{*}{ Risks factors } & Study group & Control group & \multirow{2}{*}{ P-value } \\
\cline { 2 - 3 } & $\mathbf{N = 9 3 ( \boldsymbol { P } \% )}$ & $\mathbf{N = 1 5 9 ( P \% )}$ & \\
\hline Urban residence & $47(50)^{*}$ & $82(51)^{*}$ & $>0.05$ \\
Rural residence & $42(45)$ & $38(24)$ & $<\mathbf{0 . 0 0 1}$ \\
Homelessness & $4(4)$ & $39(25)$ & $<\mathbf{0 . 0 5}$ \\
Migration & $7(7)$ & $16(10)$ & $>0.05$ \\
History of detention & 0 & $2(1)$ & $>0.05$ \\
Close contact & $5(5)$ & $15(9)$ & $>0.05$ \\
Associated diseases & $71(76)$ & $24(15)$ & $<\mathbf{0 . 0 0 1}$ \\
(excluding diabetes) & & &
\end{tabular}

Note: the applied statistical test: the paired sample T-test. *Absolute numbers and percentages per column (in brackets).

The study investigated the applied method for the screening of TB. Symptomatic screening performed by the general medical staff of the primary healthcare sector detected in a higher proportion the patients from the CG $68(43 \%)$ compared with the SG, $20(21 \%)$ cases. The active screening, on the basis of the radiological investigation of the high risk groups was used in a higher proportion in the SG $49(53 \%)$ compared with $34(21 \%)$ cases from the CG. The rate of the patients detected through the active screening realized by the medical specialists at the level of the specialized healthcare was statistically higher in the SG-26 (28\%) compared with the CG - $10(6 \%)$. However, the rate of the patients detected through the symptomatic screening realized by the medical specialists at the level of the specialized healthcare was insignificantly higher in the SG-23 (25\%) compared with the CG - 24 (15\%). Only patients from the CG came directly to the specialized TB hospital for avoiding the screening procedures of the primary healthcare facilities. Thus, the specialized 
therapeutic intervention was more accessible for diabetic patients and the direct addressing to the TB hospital was preferred way for patients without glycemic disorders.

Evaluating the disease history, it was established that patients treated for TB before actual disease was diagnosed statistically predominated in the SG - 32 (34\%) compared with $31(19 \%)$ patients in the CG. Analyzing those categories was observed that the patients which started the treatment after more than 2 months of interruption statistically predominated in the SG -16 $(17 \%)$ compared with $9(6 \%)$ in the CG. The major cause of the treatment interruption in the SG was the adverse drug reactions and in the $\mathrm{CG}$ the patients' incompliance. A similar rate was observed concerning the relapse $-8(9 \%)$ cases in the SG and $14(9 \%)$ cases in the CG, as well the treatment failure - 7 (7\%) cases in the SG compared with 8 $(9 \%)$ cases in the CG.

The assessment of the laboratory results identified a similar rate of the patients that were positive on ZiehlNeelson staining and conventional cultures. The MDRTB was diagnosed through GeneXpert MTB/RIF assay in $20(23 \%)$ cases in the SG and $44(28 \%)$ cases in the $\mathrm{CG}$ and was confirmed through the conventional cultures (Table IV).

According to the including criteria, all patients from both groups were diagnosed with pulmonary TB, 6 patients from the SG (6\%) were diagnosed with an associated extrapulmonary localization and a generalized form was present in 1 case (1\%). No extrapulmonary or generalized forms were diagnosed in the CG. The distribution of the patients according to the clinical and radiological criteria resulted in similar rates of the patients diagnosed with infiltrative TB and the predomination of the fibro-cavernous TB in the SG. All patients with chronic evolution had been treated for TB, before actual episode was diagnosed.

The assessment of the Body Mass Index (BMI) as an indicator of the nutritional status identified that in the
SG 32 (34\%) patients were malnourished (BMI $<19 \mathrm{~kg} /$ $\mathrm{m}^{2}$ ) and $45(48 \%)$ patients were undernourished (BMI 19$21 \mathrm{~kg} / \mathrm{m}^{2}$ ). In the CG a higher rate of the patients were malnourished - 69 (43\%) and a lower proportion of cases were undernourished - $61(38 \%)$. The rate of patients with normal weight was similar in both groups: $17 \%$ (16 cases) in the SG compared with $18 \%$ (29 cases) in the CG. Before TB was diagnosed, BMI had decreased to less than $21 \mathrm{~kg} /$ $\mathrm{m}^{2}$ in $77(83 \%)$ cases in the SG and in $130(81 \%)$ cases in the CG. Comparing the selected groups, no statistical difference was identified regarding the values of the BMI.

The standard treatment for drug-susceptible TB according to WHO recommendations is performed in the Republic of Moldova since 2000 and selected patients were treated standardized. The new cases from both groups with drug-susceptible TB were treated during 6 months and previously treated 8 months. The average duration of the hospitalization of the new cases was 65 days in the SG and 60 days in the CG and of the previously treated cases 98 days in the $\mathrm{SG}$ and 92 days in the CG.

The standard treatment for the drug-susceptible TB was applied in $73(78 \%)$ cases of the SG and 115 (72\%) cases of the CG. It included the following drugs: Isoniazid, Rifampicin, Pyrazinamide and Streptomycin or Ethambutol at a dose adjusted to the weight assessed at the onset of the intensive phase and at the onset of the continuation phase of the treatment. The standard treatment for MDR-TB was provided in $20(23 \%)$ patients of the SG and $44(28 \%)$ patients of the CG and included the $2^{\text {nd }}$ line antituberculous drugs recommended according to the drug susceptibility test. The standard treatment for MDRTB included injectable antibiotics - aminoglycosides (Amikacin or Capreomycin) and orally administrated anti-tuberculous drugs: fluoroquinolones (Levofloxacin or Moxifloxacin), Ethionamide or Prothionamide, Paraaminosalicylic acid and Cycloserine. New drugs, such as Delamanid and Bedaquiline, were indicated in specific cases of MDR-TB.

Table IV. Methods of detection, case-types and microbiological features.

\begin{tabular}{|c|c|c|c|c|}
\hline \multirow{2}{*}{ Reference } & \multirow{2}{*}{ Case characteristics } & Study group & Control group & \multirow{2}{*}{ P-value } \\
\hline & & $\mathrm{N}=93(P \%)$ & $\mathrm{N}=159(P \%)$ & \\
\hline \multirow{2}{*}{$\begin{array}{l}\text { Primary health care } \\
\text { providers }\end{array}$} & Detected by GPs - symptomatic patients & $20(21)^{*}$ & $68(43)^{*}$ & $<0.001$ \\
\hline & Detected by GPs - screening of HRG & $23(25)$ & $24(15)$ & $>0.05$ \\
\hline \multirow{3}{*}{$\begin{array}{l}\text { Specialized health care } \\
\text { level }\end{array}$} & Detected by SP - symptomatic patients & $24(26)$ & $35(22)$ & $>0.05$ \\
\hline & Detected by SP - screening of HRG & $26(28)$ & $10(6)$ & $<0.001$ \\
\hline & Addressed directly to the specialized TB hospital & 0 & $22(14)$ & $<0.001$ \\
\hline \multirow{2}{*}{ Case type } & New cases & $61(66)$ & $128(80)$ & $<0.001$ \\
\hline & Previously treated for TB & $32(34)$ & $31(19)$ & $<0.001$ \\
\hline \multirow{3}{*}{ Microbiological results } & Positive on AFB microscopy & $38(44)$ & $79(50)$ & $>0.05$ \\
\hline & Positive on conventional cultures & $46(53)$ & 94 (59) & $>0.05$ \\
\hline & MDR-TB & $20(23)$ & $44(28)$ & $>0.05$ \\
\hline
\end{tabular}

Note: the applied statistical test: the paired sample T-test; GP-general practitioner, SP-specialist, HRG - high risk group.

*Absolute numbers and percentages per column (in brackets). 
Table V. Clinical forms, administered treatment and effectiveness.

\begin{tabular}{|c|c|c|c|c|}
\hline \multirow{2}{*}{ Reference } & \multirow{2}{*}{$\begin{array}{l}\text { Clinical forms, treatment, } \\
\text { outcome }\end{array}$} & Study group & Control group & \multirow{2}{*}{ P-value } \\
\hline & & $\mathrm{N}=93(\mathrm{P} \%)$ & $\mathrm{N}=159(P \%)$ & \\
\hline \multirow{3}{*}{$\begin{array}{l}\text { Clinical forms of } \\
\text { pulmonary TB }\end{array}$} & IPT & $85(91)^{*}$ & $144(90)^{*}$ & $>0.05$ \\
\hline & DPT & $1(1)$ & $14(9)$ & $<0.001$ \\
\hline & FCV & $7(8)$ & $1(1)$ & $<0.001$ \\
\hline \multirow{3}{*}{$\begin{array}{l}\text { Anti-tuberculous } \\
\text { treatment }\end{array}$} & $\begin{array}{c}\text { First-line drugs } \\
\text { (for drug susceptible TB) }\end{array}$ & $73(78)$ & $115(72)$ & $>0.05$ \\
\hline & $\begin{array}{l}\text { Second-line drugs } \\
\text { (for MDR-TB) }\end{array}$ & $20(23)$ & $44(28)$ & $>0.05$ \\
\hline & Adverse drug reactions & $18(19)$ & $7(4)$ & $<0.001$ \\
\hline \multirow{4}{*}{$\begin{array}{l}\text { Treatment } \\
\text { effectiveness }\end{array}$} & Treatment success & $66(71)$ & $140(88)$ & $<0.001$ \\
\hline & Treatment failure & $7(7)$ & $6(4)$ & $>0.05$ \\
\hline & Lost to follow-up / treatment interrupted & $6(6)$ & $12(7)$ & $>0.05$ \\
\hline & Died & $14(15)$ & $1(1)$ & $<0.001$ \\
\hline
\end{tabular}

Note: the applied statistical test: the paired sample T-test; IPT - infiltrative pulmonary TB, DPT - disseminated pulmonary TB, FCVT fibro-cavernous TB.*Absolute numbers and percentages per column (in brackets).

The treatment success rate, which included cured and those who completed the treatment was statistically higher in the CG - 140 (88\%) compared with 66 (71\%) patients in the SG. The death rate was statistically higher in the SG - 14 (15\%) compared with $1(1 \%)$ in the CG. A similar rate of patients in both groups was lost to follow-up or failed the treatment.

The rate of the adverse anti-tuberculous drugs reactions was statistically higher in the SG 18 (19\%) compared with $7(4 \%)$ cases in the CG. Evaluating the adverse drugs reactions, was established that toxic types were more frequently diagnosed in the SG - $12(13 \%)$ compared with $5(3 \%)$ cases in the CG. In all cases were related to the gastro-intestinal tract. The alergic types were diagnosed in $6(6 \%)$ cases of the SG and $2(1 \%)$ cases in the CG (Table V).

The most commonly used anti-diabetic treatment was a combination of short- and intermediate-acting insulin in $32(34 \%)$ patients, intermediate-acting insulin only in $19(20 \%)$ cases, and short-acting insulin in $5(5 \%)$ cases. A combination of insulin and oral antidiabetic drugs (Metformin) was established in $12(13 \%)$ cases. In 25 (27\%) cases only oral antidiabetic treatment (monotherapy with Metformin or a combination of Metformin and sulfonylureas) was recommended. The FVPG was monitored weekly in all patients during the hospitalization period. In 22 (24\%) patients the FVPG exceeded the normal threshold despite the antidiabetic treatment and diabetes diet. The patients were advised by the hospital dietitian about the methods of the metabolic imbalance prevention, the type of diet with the amounts of carbohydrates determined according to age and body weight. The diet based on slow-releasing glucose foods, such as nondigestible fiber-rich foods (fresh vegetables, whole grains, and beans) was recommended. The patients were advised to decrease the consumption of saturated fats and increase the consumption of unsaturated fats (vegetable oils), fish and white meat. They received the recommendations to decrease the consumption of salt, refined sugar products, and to stop smoking and alcohol drinking. The patients were informed that the failure to follow the dietitian's and endocrinologist's recommendations could lead to the onset of acute complications and death.

\section{Discussion}

Our research is among the few works in the Republic of Moldova on an important topic related to TB and diabetes. In our study, type 2 diabetes was diagnosed more often, even if the patients with type 1 diabetes are more vulnerable to infection and occurrence of tuberculosis [20]. The convergence of two epidemics, TB and DM increased the number of patients with the type 2 of DM, fact demonstrated by a recent large meta-analysis [21]. Our research established that TB was diagnosed more often in patients with complications, in which the FVPG exceeded the normal limit. In the literature many studies established that patients with uncontrolled blood glucose level have high risk for occurrence of TB [11-15,23,25]. Although men are significantly more at a risk for contracting and dying from TB than women, our research established a similar distribution of the diabetic patients in groups according to the sex. It was recognized that the age groups with the greatest percentage of notified TB cases are 15-24 and 25-34 years [1-3]. Our research concluded that diabetic patients are often older 55 years. This is because the adults aged between 45 to 64 years were diagnosed with the type $2 \mathrm{DM}$, which prevailed in our study.

Our study and the literature established that the social and economic vulnerability constituted a risk factor for TB in patients with DM [6,7,11-16,21,23,25]. However, 
our research established a high rate of the diabetic patients with free access to the screening procedures, due to specific conditions, like have been retired or disabled. The low rate of TB detected within the investigation of the tuberculous clusters in diabetic and non-diabetic groups, reflected poor quality of the contacts' examination. We confirmed that the annual radiologic investigation of diabetic patients was an efficient way for TB detection. The result has been sustained by other study [22]. Even the active radiological screening was routinely used, the TB with chronic evolution, such as fibro-cavernous type was more often diagnosed in diabetic patients. The literature explained the effect of the uncontrolled glucose level in the blood on the releasing of the pro-inflammatory cytokines and occurrence of fibrotic changes in the lung tissue [7]. Furthermore, in our research the diabetic patients with longlasting disease had been diagnosed with the relapse of TB after a successfully completion of the treatment or started the treatment again after interruption. High rate of the TB recurrence and recovered after the lost to follow-up were showed by other researches [11-13,24]. The main causes contributing to the recurrence a new episode of TB are: long duration for sputum-smear conversion from positive in negative and short duration of the standardized antituberculous treatment, high rate of MDR-TB and adverse drug reactions, detection in late stage and dysfunction of the immunity [26].

The study of the adverse drug reactions in patients with DM found that most commonly diagnosed were toxic events related to the gastrointestinal system. According to the literature, in patients with DM the most frequent adverse drug reaction were identified related of gastrointestinal tract and endocrine system (glycemic disorders) $[17,18]$.

The standard treatment for drug-susceptible and MDR-TB was applied in a similar proportion in both groups. However, there are papers demonstrating a higher rate of drug resistance in diabetic patients [16,19,21]. The Republic of Moldova ranks among the countries with a high prevalence of drug resistance, which explained that every fourth patient in both selected groups was microbiologically confirmed with MDR-TB. The antituberculous treatment effectiveness was considerably lower in the diabetic group, through a higher mortality rate compared with non-diabetic patients. The obtained results were similar to other studies, that identified a high rate of death and treatment failure [6,7,11-17,23,24]. All patients with DM received an individualized treatment, but in every fourth the glycemic control was not achieved. Deficiencies in the glucose control had been reported during the antituberculous treatment in different researches, mostly due to the toxicity of the anti-TB drugs [11-18].

To the best of our knowledge, this study is the first to identify the characteristic features of the patients diagnosed with TB and DM in a high burden country and to clarify the main risk factors, which contributed to the occurrence of TB. Furthermore, we identified the methods of detection of TB and conditions contributing to the disease outcome. The limitations of this study should be also considered. The small number of patients included, as a result of the inclusion criteria, make the observation applicable only to the epidemiological situation in the Republic of Moldova. Further studies on a larger scale are needed to establish the conditions with high impact on the outcome of both diseases: DM and TB.

\section{Conclusions}

In clinical practice, the main features of diabetic patients with risk for TB were age older 55 years and social-economical vulnerability. Our study identified that the anti-tuberculous treatment outcome was diminished by glycemic disorders (hyperglycemia) and related complications, history of the previous anti-tuberculous treatment and adverse drug reactions. Thus, we concluded that a closed follow-up through the active screening should be performed regularly in all patients with glycemic disorders. The high rate of the patients with previous antituberculous treatment and the increased rate of those who developed adverse drug reactions demonstrated that the individualized therapeutic approach of TB could improve the treatment effectiveness.

\section{References}

1. World Health Organization. Global tuberculosis report 2018. https://apps.who.int/iris/handle/10665/274453

2. Centrul National de Management in Sanatate [National Center for Health Management]. Indicatori preliminari privind sănătatea populaţiei şi activitatea instituţiilor medico-sanitare pe anii 2016-2017 [Preliminary indices on the health of the population and activity of the medical sanitary institutions for 2016-2017]. 2018. Available from: http://old2.ms.gov.md/?q=rapoarte/indicatori-preliminariformat-prescurtat-privind-sanatatea-populatiei-si-activitatea

3. World Health Organization. The End TB Strategy. Available from: https://www.who.int/tb/strategy/End_TB_Strategy. pdf

4. United Nations. Sustainable Development Goals. Available from: https://www.un.org/sustainabledevelopment/wpcontent/uploads/2019/01/SDG_Guidelines_January_2019. pdf

5. American Diabetes Association. Standards of medical care in diabetes. Diabetes Care. 2018 Jan; 41(1): (Suppl 1):S73-S85. doi: $10.2337 / \mathrm{dc} 18-\mathrm{S} 008$

6. Baghaei P, Marjani M, Javanmard P, Tabarsi P, Masjedi MR. Diabetes mellitus and tuberculosis facts and controversies. J Diabetes Metab Disord. 2013;12:58.

7. Restrepo BI, Fisher-Hoch SP, Pino PA, Salinas A, Rahbar $\mathrm{MH}$, Mora F, et al. Tuberculosis in poorly controlled type 2 diabetes: altered cytokine expression in peripheral white blood cells. Clin Infect Dis. 2008;47:634-641. 
8. Ministerul Sănătății [Ministry of Health]. Protocol Clinic Național [Clinical National Protocol]. Diabetul zaharat necomplicat [Non-complicated diabetes mellitus]. 2017. Available from: http://89.32.227.76/_files/15516-PCN33\%2520DZ.pdf

9. Ministerul Sănătății [Ministry of Health]. Protocol Clinic Național [Clinical National Protocol]. Tuberculoza la adulți [Tuberculosis in adults]. 2017. Available from: http://89.32.227.76/files/15189tuberculoza\%2520la\%2520adult.pdf

10. World Health Organization. Treatment of tuberculosis: guidelines, $4^{\text {th }}$ edition. Available from: https://apps.who.int/ iris/bitstream/handle/10665/44165/9789241547833_eng. pdf? sequence $=1$

11. Baker MA, Harries AD, Jeon CY, Hart JE, Kapur A, Lönnroth $\mathrm{K}$, et al. The impact of diabetes on tuberculosis treatment outcomes: a systematic review. BMC Med. 2011;9:81.

12. Chang JT, Dou HY, Yen CL, Wu YH, Huang RM, Lin $\mathrm{HJ}$, et al. Effect of type 2 diabetes mellitus on the clinical severity and treatment outcome in patients with pulmonary tuberculosis: a potential role in the emergence of multidrugresistance. J Formos Med Assoc. 2011;110:372-381.

13. Siddiqui AN, Khayyam KU, Sharma M. Effect of Diabetes Mellitus on Tuberculosis Treatment Outcome and Adverse Reactions in Patients Receiving Directly Observed Treatment Strategy in India: A Prospective Study. Biomed Res Int. 2016;2016:7273935.

14. John NM, George JM, Poornachary NM. Effect of antitubercular drugs and impact of glycemic status in patients with tuberculosis and concomitant diabetic-tuberculosis patients. Ind. J. Pharm. Pract. 2017; 10(2): 125-128. doi:10.5530/ijopp.10.2.25

15. Harries AD, Kumar AM, Satyanarayana S, Lin Y, Zachariah R, Lönnroth K, et al. Diabetes mellitus and tuberculosis: programmatic management issues. Int $\mathrm{J}$ Tuberc Lung Dis. 2015;19:879-886.

16. Muñoz-Torrico M, Caminero-Luna J, Migliori GB, D'Ambrosio L, Carrillo-Alduenda JL, Villareal-Velarde H, et al. Diabetes is Associated with Severe Adverse Events in Multidrug-Resistant Tuberculosis. Arch Bronconeumol. 2017;53:245-250.

17. Tornio A, Niemi M, Neuvonen PJ, Backman JT. Drug interactions with oral antidiabetic agents: pharmacokinetic mechanisms and clinical implications. Trends Pharmacol Sci. 2012;33:312-322.

18. Ruslami R, Nijland HM, Adhiarta IG, Kariadi SH, Alisjahbana B, Aarnoutse RE, et al. Pharmacokinetics of antituberculosis drugs in pulmonary tuberculosis patients with type 2 diabetes. Antimicrob Agents Chemother. 2010;54:1068-1074.

19. Lesnic E, Malic A, Kulcitkaia S, Niguleanu R, Jucov A, Gutu-Grecu M, et al. The predictors of pulmonary tuberculosis in Xpert MBT/Rif positive and resistant assay patients with diabetes mellitus. The Moldovan Medical Journal. 2018;61:23-29. doi: 10.5281/zenodo.1299008

20. Webb EA, Hesseling AC, Schaaf HS, Gie RP, Lombard CJ, Spitaels A, et High prevalence of Mycobacterium tuberculosis infection and disease in children and adolescents with type 1 diabetes mellitus. Int J Tuberc Lung Dis. 2009;13:868-874.

21. Dooley KE, Chaisson RE. Tuberculosis and diabetes mellitus: convergence of two epidemics. Lancet Infect Dis. 2009;9:737-746.

22. Lesnic E, Malic A, Lupu E. Aportul screening-ului în depistarea tuberculozei la pacienţii cu diabet zaharat [The impact of the screening in the detection of tuberculosis in patients with diabetes mellitus]. Sănătate Publică, Economie şi Managementul în Medicină [Public Health, Economy and Management in Medicine]. 2018, 1-2(75-76): 2933. Available on http://revistaspemm.md/wp-content/ uploads/2019/05/1-275-762018.pdf

23. Workneh MH, Bjune GA, Yimer SA. Prevalence and associated factors of tuberculosis and diabetes mellitus comorbidity: A systematic review. PLoS One. 2017; 12:e0175925.

24. Shewade HD, Jeyashree K, Mahajan P, Shah AN, Kirubakaran R, Rao R, et al. Effect of glycemic control and type of diabetes treatment on unsuccessful TB treatment outcomes among people with TB-Diabetes: A systematic review. PLoS One. 2017; 12:e0186697.

25. Yorke E, Atiase Y, Akpalu J, Sarfo-Kantanka O, Boima V, Dey ID. The Bidirectional Relationship between Tuberculosis and Diabetes. Tuberc Res Treat. 2017;2017:1702578.

26. Restrepo BI. Diabetes and Tuberculosis. Microbiol Spectr. 2016;4:10.1128/microbiolspec.TNMI7-0023-2016. 